\title{
An Investigation of Documents from the World Wide Web
}

\author{
Allison Woodruff \\ Paul M. Aoki \\ Eric Brewer \\ Paul Gauthier \\ Lawrence A. Rowe \\ Computer Science Division \\ University of California at Berkeley \\ Berkeley, CA 94720-1776 \\ email: \{woodruff,aoki,brewer,gauthier,rowe\}@cs.berkeley.edu
}

\begin{abstract}
:
We report on our examination of pages from the World Wide Web. We have analyzed data collected by the Inktomi Web crawler (this data currently comprises over 2.6 million HTML documents). We have examined many characteristics of these documents, including: document size; number and types of tags, attributes, file extensions, protocols, and ports; the number of in-links; and the ratio of document size to the number of tags and attributes. For a more limited set of documents, we have examined the following: the number and types of syntax errors and readability scores. These data have been aggregated to create a number of ranked lists, e.g., the ten most-used tags, the ten most common HTML errors.
\end{abstract}

\section{Keywords:}

HTML, statistics, tools, World Wide Web.
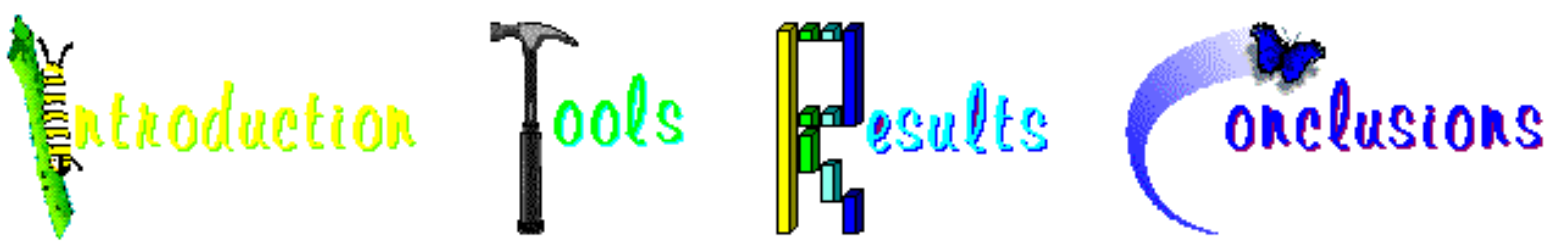

\section{Introduction}

We report the results of an extensive analysis of HTML documents from the World Wide Web. Our data set, collected by the Inktomi Web crawler, currently comprises over 2.6 million HTML documents. We present a broad range of statistics pertaining to these pages.

Such an analysis of the content of HTML documents is of interest for several reasons:

- Evolution of HTML. Unused features and extensions that do not achieve a reasonable level of acceptance should be deprecated and, eventually, eliminated. This prevents the accretion of useless language features. 
- Improving Web content. Widespread awareness of poor natural and markup language usage will promote the spread of helpful tools and practices.

- Control of HTML. The marketplace perceives the relative ability of vendors to force acceptance of new, non-standard language extensions as market "strength." Understanding the true acceptance level of such extensions can help fight vendor disinformation.

- Sociological insights. Many interesting sociological observations may be derived from the content of Web pages.

Despite these motivations, however, previous studies relating to the Web have either focused on other topics or have been limited in scope. The most closely related work includes:

- User studies. User surveys [COMM95, PITK94b, PITK95a, PITK95b, RISS95, YAHO95] and browser usage studies [CATL95, PITK94a] have become very common. Such studies focus on high-level user issues (e.g., choice of software, available connectivity) and low-level user-browser interaction (e.g., use of the back button). The information extracted, though valuable, is wholly user-centric.

- Content analyses of small data sets. There have been some attempts to perform simple analyses of the content of the Web. For example, the original Lycos project at Carnegie Mellon University's Center for Machine Translation [MAUL94] tracked a number of interesting statistics while their data set was relatively small. These included:

$O$ content of title and headings

O 100 top keywords and first 20 lines

o word frequency count

O file size (bytes, words)

O URL types

O most-linked-to URLs

- Structural analysis. The CMU Lycos project generated at least one complete graph of their data set. The project's commercial successor, Lycos, Inc., now tracks the 250 most-linked-to sites as a side-effect of their indexing [LYCO95]. Other projects have focused on (graph-oriented) structural analysis as well. These include several Web visualization systems (e.g., Webspace [CHI95] and the Navigational View Builder [MUKH95]). For the most part, such visualization has been very small-scale and limited in scope. More sophisticated analyses are possible, combining both structural analysis and semantic modelling. A project at Xerox PARC [PIRO95] is conducting such analyses over small data sets.

To complement the above work, we have conducted a large-scale investigation of the content of HTML documents from the Web. The remainder of this paper is structured as follows. First, we describe the tools we used to perform our study. We next discuss the scope of our study and our results. Finally, we present some lessons learned and possible future directions.

\section{Tools}

The tools used to perform the data collection and data analysis for this study represent the integration of software from a variety of sources. Specifically, we have developed or adapted software to perform the following tasks:

- Web Data Collection 
- Data Extraction and Manipulation

- Natural (English) Language Analysis

- Markup (HTML) Language Analysis

We discuss each set of tools in turn.

\section{Web Data Collection}

The Inktomi research project at Berkeley, consisting of Prof. Eric Brewer and graduate student Paul Gauthier, conducts research in the construction of scalable Web servers using parallel processing technology. To date, the project has produced two major software components: a parallel Web crawler and a parallel Web index search engine. In this paper, where we mention Inktomi, it may be assumed that we refer to the crawler.

The data presented in this study comes entirely from Inktomi. The high speed of the crawler enables us, for the first time, to consider taking "snapshots" of the Web and analyzing them. As of this writing, the Inktomi team has crawled twice. The first set of runs, from July to October 1995, collected 1.3 million unique HTML documents. The second set of runs, in November 1995, collected 2.6 million unique HTML documents.

\section{HTML Data Extraction and Manipulation: libink}

Although toolkits such as the W3C Reference Library [FRYS94] already exist for manipulating HTML and HTTP objects, we have developed our own special-purpose library, libink. This was necessitated by the fact that our performance and functionality needs were very different from those of the other toolkit developers.

libink consists of four major subcomponents:

- HTML parser. libink contains a simple flex-based HTML scanner. We found existing parsers too slow (especially true in the case of parsers written in scripting languages) or difficult to modify. The libink scanner is small, enabling us to make it both fast and relatively robust, as well as highly configurable. Like the W3C SGML/HTML lexical analyzer [CONN95], our scanner uses a callback interface to handle various events (e.g., recognition of a tag and its attributes). The W3C lexical analyzer, however, is not configurable.

- URL parser. The URL parser, unlike many freely-available implementations, conforms to RFC 1808 [FIEL95].

- Domain name service (DNS) translation and caching. We use Internet addresses to reduce hostname aliasing in our data. To speed up the lookup process, we provide a wrapper around the standard name service routines that caches all URL hostnames.

- General hash table services. The various lookup tables on which libink relies sometimes exceed the capacity of a single machine's physical memory. Therefore, in addition to in-memory hash tables, libink provides interfaces to striped on-disk hash tables (using GNU DBM) as well as hash-partitioned distributed hash tables (using ONC RPC). The distributed hash tables support $1 \mathrm{~ms}$ turnaround on hash table lookups, which is far better than the $20-30 \mathrm{~ms}$ required to fetch a hash table page from secondary storage. 


\section{Natural Language Analysis: style}

We scored English language documents using the standard UNIX style program [CHER81]. style reports a variety of statistical properties of each document, such as the average sentence length and the number of complex sentences. It also scores the document using four readability metrics. These metrics indicate the nominal educational (grade) level a reader would need to understand the document.

Since most HTML documents do not conform to an internationalization standard, we applied heuristics to screen out non-English documents. We filtered out documents that contained any character with the high bit set (indicating a non-ASCII character set) or containing character sequences indicating known encodings (such as the Shift-JIS encoding of the Japanese character set).

\section{Markup Language Analysis: weblint}

We scored documents using weblint [BOWE96], an analogue to the standard UNIX lint utility, written in Perl. We modified weblint to report the classes of errors in a document rather than a line-by-line analysis.

\section{Results}

We examined over 2.6 million HTML documents collected by the Inktomi crawler in November of 1995. Although Inktomi occasionally downloads non-HTML documents, the results presented reflect only HTML documents. (For example, we filtered out all binary files, such as images.) Furthermore, because Inktomi implements the Robot Exclusion Standard, the contents of automated databases which follow the standard (e.g., genome data sets) have also been excluded. The distribution of the documents in the data set by domain appears in Table 1.

\begin{tabular}{|l||r||r||}
\hline Domain & \# of HTML Documents & \% of Total \\
\hline \hline other & 1064318 & $41 \%$ \\
\hline \hline com & 516709 & $20 \%$ \\
\hline \hline edu & 698616 & $27 \%$ \\
\hline \hline gov & 117125 & $4 \%$ \\
\hline \hline net & 113595 & $4 \%$ \\
\hline \hline mil & 14734 & $1 \%$ \\
\hline \hline org & 89939 & $3 \%$ \\
\hline total & $\mathbf{2 6 1 5 0 3 6}$ & $\mathbf{1 0 0 \%}$ \\
\hline
\end{tabular}

Table 1: Documents Studied by Domain

Here, "other" includes all domains other than the given top-level domains. For example, "other" contains all non-US top-level domains (such as Germany’s . de).

We analyzed a variety of properties of these documents. In this paper, we present results on the following: 
- Document Size

- Tag/Size Ratio

- Tag Usage

- Attribute Usage

- Browser-specific Extension Usage

- Port Usage

- Protocols Used in Child URLs

- File Types Used in Child URLs

- Number of In-links

- Readability

- Syntax Errors

\section{Document Size}

After all markup had been extracted, the size of each HTML document was measured. For the entire data set, the mean size was $4.4 \mathrm{~KB}$, the median size was $2.0 \mathrm{~KB}$, and the maximum size was $1.6 \mathrm{MB}$.

Figure 1 presents different views of the size distribution. On first inspection, this distribution appears to be exponential (the magenta line represents the location of the mean). However, further zooming indicates a curve before the distribution begins to taper off. The final graph in Figure 1 contains a semilog plot of the same data (in which the sizes are plotted logarithmically and the number of documents is plotted arithmetically).
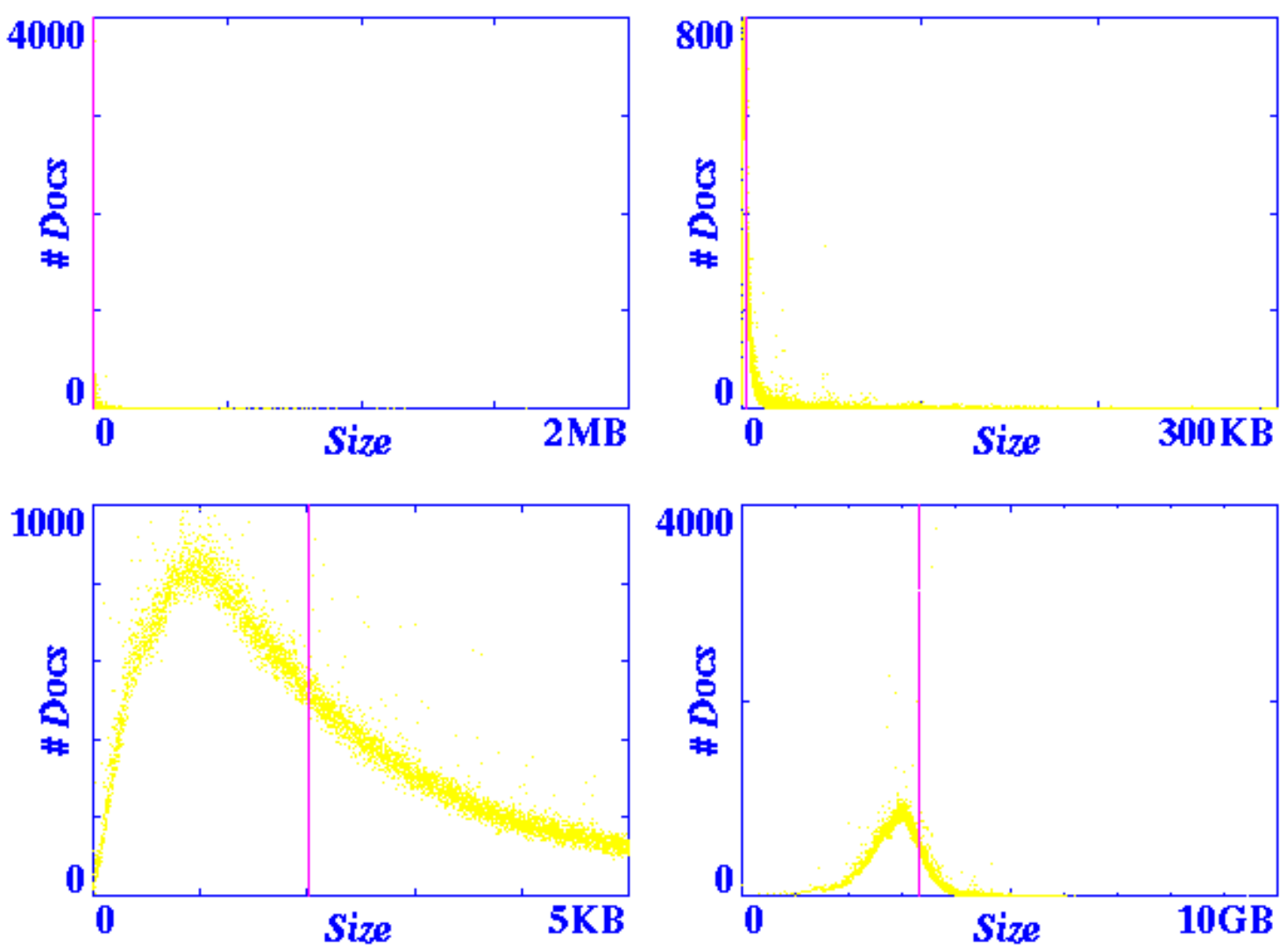

Figure 1: Size Distribution 
These simple size distribution plots proved to be very useful in detecting several problems with the data set. Many of the outliers were caused by one of two major classes of errors:

- Problematic URLs: when faced with incorrect URLs that contain valid prefixes, some HTTP servers return the file matching the valid prefix. For example, the data set contains hundreds of documents with URLs of the form http://bazaar.com/underground2.html/..., all of which are identical to http://bazaar.com/underground2.html. There does not appear to be a general way for a client program (such as a crawler) to differentiate this situation from a site containing a large number of identical files.

- CGI Error Responses: some of the most popular CGI programs, such as NCSA imagemap and CERN hT Image, report errors with messages containing HTTP status " 200 ”' (success). Because the image map programs all happen to return fixed error messages, we were able to detect and eliminate those particular messages, but there (again) does not appear to be any general way for a client to distinguish " 200 ” error messages from valid documents.

\section{Tag/Size Ratio}

For each document we examined the ratio of the total number of tags to its size. Figure 2 contains the results. An interesting pattern emerges - rays radiating out from the origin, indicating a number of documents with constant tag/size ratios. One such ray is indicated by the green ellipse. We examined a number of these rays and determined that they represented different versions of the same document (occurring in archives or mirrored sites). This suggests that the tag/size ratio might be used as a component of a signature for an HTML document, e.g., for purposes of copy detection.

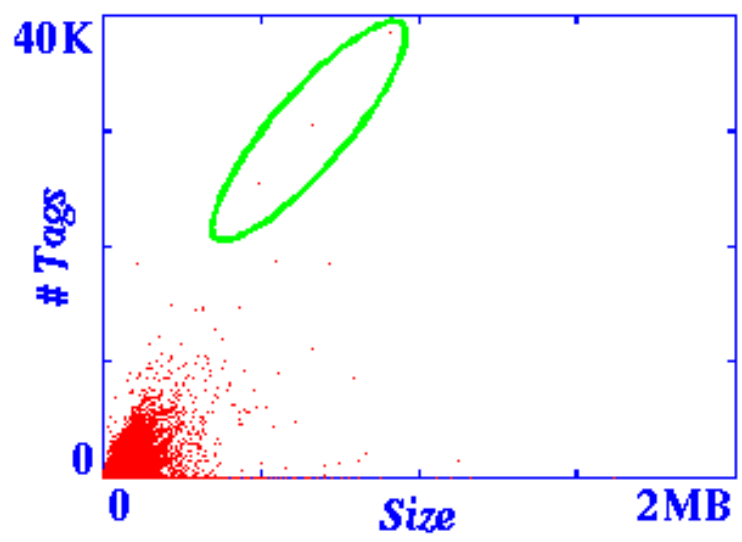

Figure 2: Tag/Size Ratio

\section{Tag Usage}

We examined the distribution of tags. We obtained a list of valid tags from the Sandia HTML Reference Manual [HANN95]. The average number of total tags per document was 71 . The average number of unique tags per document was 11.

We examined the most popular tags. The top graph of Figure 3 shows the top ten tags (ranked according to the number of documents in which the tag appeared at least once). The bottom graph indicates the average number of occurrences of the tag per document. 

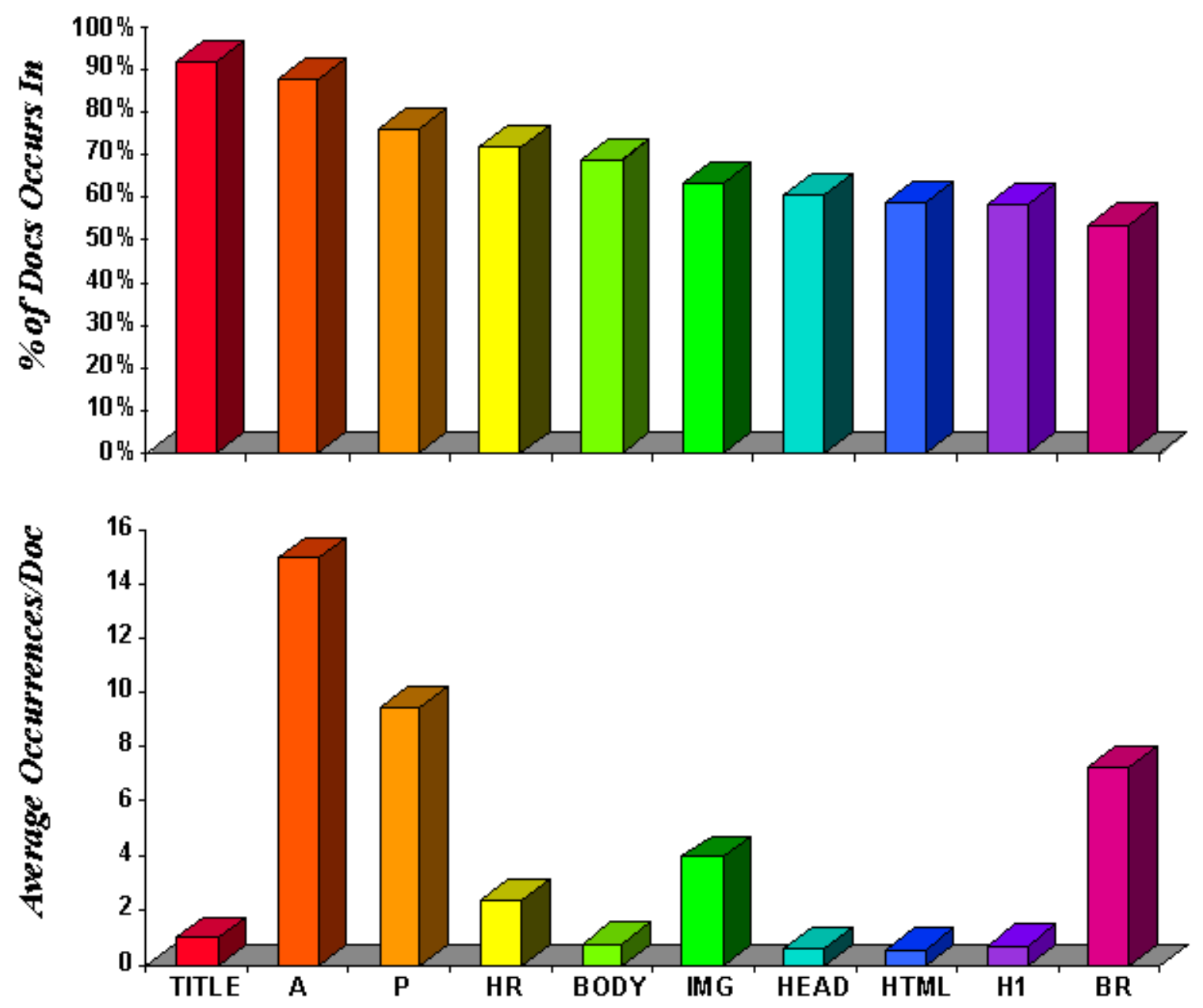

Figure 3: Ten Most-Used Tags

We also examined the least popular tags. Several tags, BDO, COLGROUP, and NOEMBED were used zero times in our data set of over 2.6 million HTML documents. A number of other tags appeared a very limited number of times.

\section{Attribute Usage}

We examined the distribution of attributes. The average number of total attributes per document was 29. The average number of unique attributes per document was 4.

We examined the most popular attributes. Figure 4 shows the top ten attributes (ranked according to the number of documents in which the attribute appeared at least once). HREF appeared an average of 14 times per document. 


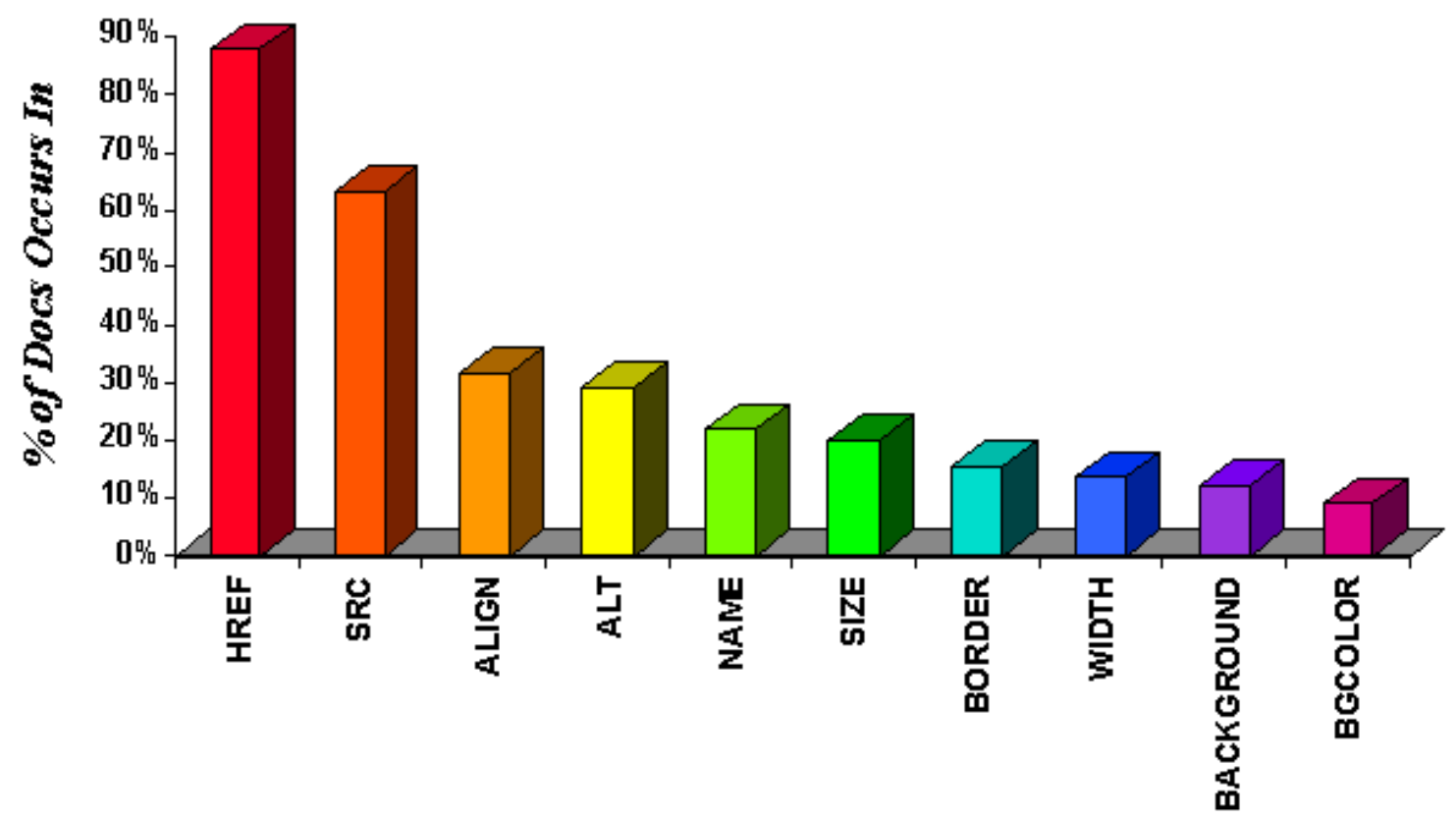

Figure 4: Ten Most-Used Attributes

We also examined the least popular attributes. Several attributes, ACCEPT-CHARSET, AXIS, CHAROFF, and CONTROLS, were used zero times in our data set of 2.6 million HTML documents. A number of other attributes appeared a very limited number of times.

\section{Browser-specific Extension Usage}

We also studied the use of browser-specific extensions. These consist of HTML features (i.e., tags or attributes) added by vendors rather than by the standards process. Here, we contrast the use of such extensions in the first Inktomi data set (1.3 million documents, collected in mid-1995) and the second Inktomi data set (2.6 million documents, collected in November 1995).

Figure 5 shows the percentage of documents in which the four most popular extensions are used. The usage of most of these features has risen dramatically, indicating wide user acceptance. Other features, such as BLINK, have not experienced such growth.

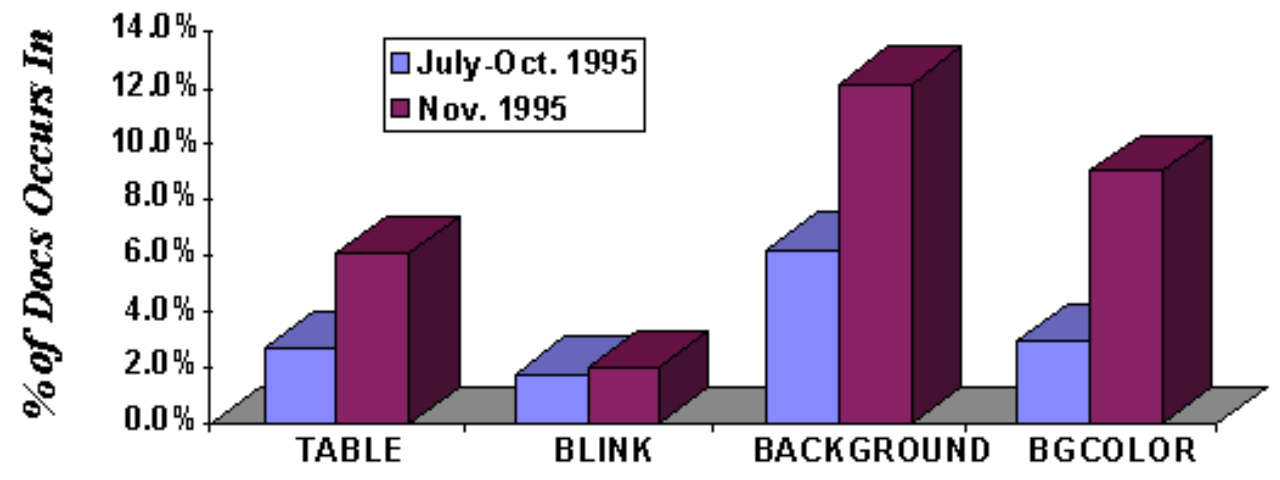

Figure 5: Browser-Specific Extensions Usage 
Figure 6 indicates the popularity of various proposals for dynamic addition of functionality to browsers. APP and APPLET support SunSoft's Java 'applet' language, DYNSRC supports VRML markup, and EMBED supports Netscape's third-party "plug-in' modules. All have enjoyed significant growth, though the oldest and most popular method (Java, first released in May 1995 [KARP95]) still has very low usage.

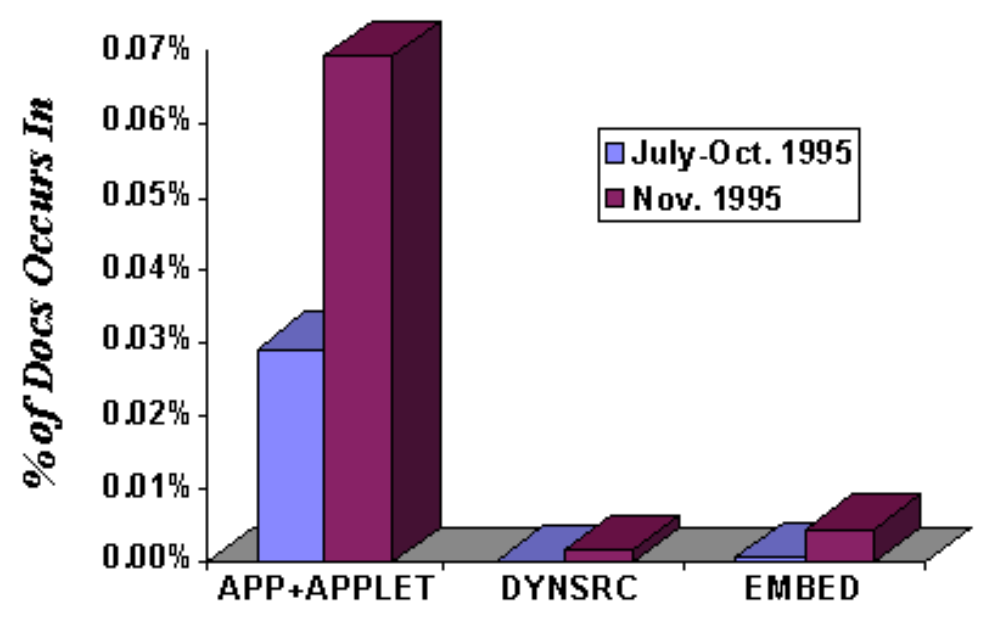

Figure 6: Browser-Specific Extensions Usage

\section{Port Usage}

For each of the HTML documents in our data set, we extracted the port number used to access the document. We analyzed the distribution of port numbers. While 418 unique ports were observed, six ports accounted for over $98 \%$ of the documents. Table 2 presents the most popular ports.

\begin{tabular}{||c||r|r|}
\hline Category & Port & \% of Docs \\
\hline \hline Standard & 80 & $93.6 \%$ \\
\hline \hline$<\mathbf{1 0 2 4}$ & 70 & $0.3 \%$ \\
\hline \hline \multirow{3}{*}{$>=\mathbf{1 0 2 4}$} & 8000 & $0.5 \%$ \\
\cline { 2 - 3 } & 8001 & $0.5 \%$ \\
\cline { 2 - 3 } & 8080 & $0.7 \%$ \\
\cline { 2 - 3 } & 8888 & $2.8 \%$ \\
\hline
\end{tabular}

Table 2: Port Usage

Port 80, the standard HTTP port, was used for approximately $94 \%$ of the documents. Port 70 (the standard Gopher port) was used for approximately $0.3 \%$ of the documents (this number is slightly lower than the $1 \%$ usage of port 70 observed in our earlier data set). We checked many of the documents being served from port 70; all the ones we examined were in fact HTML documents. Ports 8000, 8001, and 8080 , and 8888 accounted for the majority of the remaining documents. The strong preference for " 8 ", and " 80 " in the non-standard ports is presumably related to the standard port number " 80 "

\section{Protocols Used in Child URLs}


As discussed above, we extracted child URLs from all HTML documents in our data set. Figure 7 presents the distribution of protocols in this set of child URLs. By far, the most dominant protocol observed was HTTP (there were an average of 17 HTTP URLs per document).

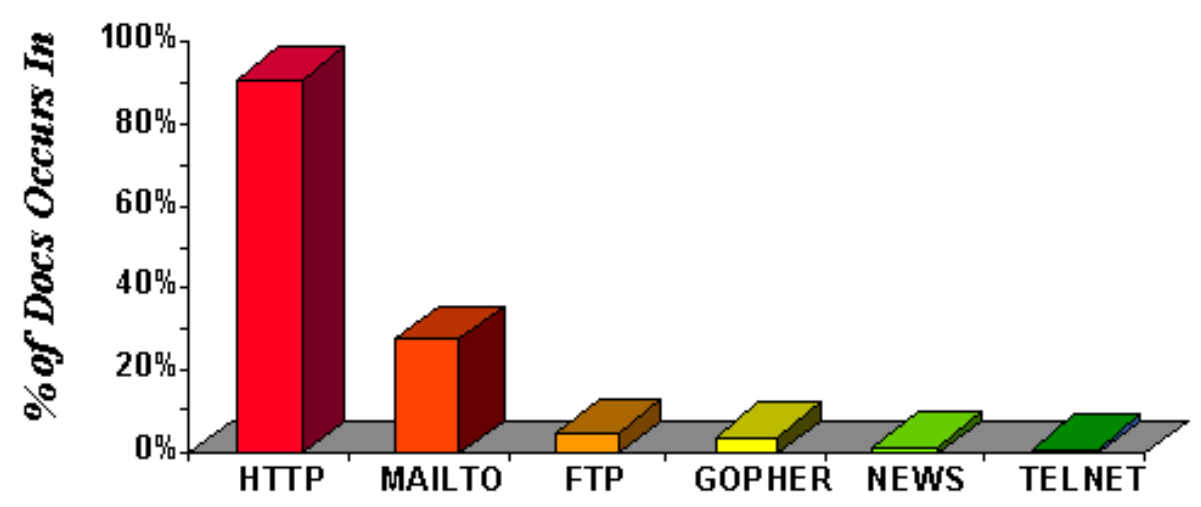

Figure 7: Protocol Usage

\section{File Types Used in Child URLs}

We also studied the distribution of file types described in the set of extracted child URLs. We inferred the file type from the file name extensions (e.g., ".gif”') found in the URL path. In Table 3, the " $\%$ of Docs" column indicates the percentage of documents which contained a file of a given type. The "\# of Occurrences" column shows the total number of extensions of the given file type that were observed. The "\# of Docs" column indicates the number of documents which contained one or more extensions of the indicated type. Note that files can be counted multiple times, e.g., file.ps.z would be counted as a file having both ".ps" and ".Z" extensions.

\begin{tabular}{||c||l||r||r||r||}
\hline \multicolumn{1}{|c||}{ Category } & \multicolumn{1}{|c|}{ Type (Extension) } & \% of Docs & \# of Occurrences & \# of Docs \\
\hline \hline \multirow{5}{*}{ Compression/Archive } & GNU zip (gz/gzip/taz/tgz) & $0.7 \%$ & 126839 & 18694 \\
\cline { 2 - 5 } & Zip (zip) & $0.7 \%$ & 157918 & 17277 \\
\cline { 2 - 5 } & compress (Z) & $0.6 \%$ & 121519 & 16857 \\
\cline { 2 - 5 } & BinHex (hqx) & $0.3 \%$ & 138259 & 7188 \\
\cline { 2 - 5 } & StuffIt (sea) & $0.1 \%$ & 5290 & 2615 \\
\cline { 2 - 5 } & LHArc (lha/lharc) & $0.0 \%$ & 20985 & 597 \\
\cline { 2 - 5 } & ARC archive (arc) & $0.0 \%$ & 432 & 129 \\
\hline
\end{tabular}




\begin{tabular}{|c|c|c|c|c|}
\hline \multirow{10}{*}{ Document } & HTML (htm/html) & $76.3 \%$ & 21982792 & 1995731 \\
\hline & text (txt) & $2.2 \%$ & 325165 & 57476 \\
\hline & PostScript (eps/ps) & $1.8 \%$ & 239949 & 46977 \\
\hline & MS Word (doc) & $0.2 \%$ & 20153 & 5959 \\
\hline & Adobe Acrobat (pdf) & $0.2 \%$ & 30640 & 5360 \\
\hline & TeX DVI (dvi) & $0.2 \%$ & 14680 & 4163 \\
\hline & Tex (tex) & $0.1 \%$ & 11998 & 2993 \\
\hline & TROFF (man/me/ms) & $0.1 \%$ & 6488 & 2191 \\
\hline & Rich Text (rtf) & $0.0 \%$ & 3921 & 1184 \\
\hline & Maker Interchange (mif) & $0.0 \%$ & 262 & 113 \\
\hline \multirow{10}{*}{ Audio } & Sun audio $(\mathrm{au})$ & $0.7 \%$ & 60405 & 18865 \\
\hline & MS WAVE (wav) & $0.3 \%$ & 24361 & 7325 \\
\hline & Audio IFF (aif/aifc/aiff) & $0.1 \%$ & 7761 & 2611 \\
\hline & MIME audio (snd) & $0.0 \%$ & 1839 & 600 \\
\hline & Amiga MOD (mod/nst) & $0.0 \%$ & 4202 & 254 \\
\hline & $\operatorname{IRCOM}(\mathrm{sf})$ & $0.0 \%$ & 353 & 161 \\
\hline & IFF (iff) & $0.0 \%$ & 322 & 47 \\
\hline & SoundBlaster (voc) & $0.0 \%$ & 122 & 27 \\
\hline & U-law (ul) & $0.0 \%$ & 21 & 19 \\
\hline & FSSD (fssd/hcom) & $0.0 \%$ & 3 & 3 \\
\hline \multirow{12}{*}{ Image } & GIF (gif) & $61.7 \%$ & 9990239 & 1614244 \\
\hline & JPEG (jpe/jpeg/jpg) & $7.8 \%$ & 811353 & 205088 \\
\hline & X bitmap (xbm) & $2.9 \%$ & 968410 & 75825 \\
\hline & TIFF (tif/tiff) & $0.2 \%$ & 22546 & 5416 \\
\hline & $\mathrm{X}$ pixmap (xpm) & $0.0 \%$ & 3448 & 814 \\
\hline & RGB (rgb) & $0.0 \%$ & 985 & 259 \\
\hline & portable pixmap (ppm) & $0.0 \%$ & 646 & 124 \\
\hline & portable graymap (pgm) & $0.0 \%$ & 219 & 78 \\
\hline & portable bitmap (pbm) & $0.0 \%$ & 114 & 70 \\
\hline & $\mathrm{X}$ window dump (xwd) & $0.0 \%$ & 277 & 66 \\
\hline & raster (ras) & $0.0 \%$ & 221 & 54 \\
\hline & portable anymap (pnm) & $0.0 \%$ & 51 & 7 \\
\hline \multirow{4}{*}{ Movie } & MPEG (mpe/mpeg/mpg) & $0.3 \%$ & 21496 & 7460 \\
\hline & QuickTime (mov/qt) & $0.2 \%$ & 15026 & 5199 \\
\hline & MS video (avi) & $0.1 \%$ & 5589 & 1742 \\
\hline & SGI (movie) & $0.0 \%$ & 538 & 313 \\
\hline
\end{tabular}

Table 3: File Type and File Name Extensions 


\section{Number of In-links}

We sorted the child URLs which we extracted according to the number of times they occurred in our data set. This showed us the most "popular" sites, as measured by the number of in-links observed. These appear in Table 4.

The in-link entries marked with $\left(^{*}\right)$ indicate sites that are highly self-referential. That is, these sites (by inspection) appear to contain a great number of links to their own top-level pages. It would probably be instructive to count only links from outside a given site.

\begin{tabular}{|c|c|c|}
\hline Site & Description & In-links \\
\hline Www.xerox.com & Xerox PARC & (*) 28188 \\
\hline www.yahoo.com & Yahoo & 19424 \\
\hline cool.infi.net & Cool Site of the Day & 19028 \\
\hline hamsterix.funet.fi & Bible (in Finnish) & $(*) 17243$ \\
\hline sundarssrv2.cern.ch & CERN preprint service & (*) 16049 \\
\hline wings.buffalo.edu & Best of the Web '94 & 14685 \\
\hline wings.buffalo.edu & U.S. Gazetteer & 14369 \\
\hline Www.ist.unige.it & Cell database & (*) 12750 \\
\hline home.netscape.com & Netscape Communications & 12081 \\
\hline www.american.recordings.com & Ultimate Band List & 11014 \\
\hline jasper.ora.com & Comprehensive TeX Archive Network & 10650 \\
\hline www.ibm.com & IBM Corp. & 10617 \\
\hline www.informatik.uni-trier.de & $\begin{array}{l}\text { Bibliography Server on Database Systems \& Logic } \\
\text { Programming }\end{array}$ & |(*) 10212 \\
\hline siva.cshl.org & wusage 3.2 (WWW usage statistics) & 9038 \\
\hline curly.cc.utexas.edu & Jane Austen's Pride \& Prejudice & (*) 8928 \\
\hline www.starwave.com & StarWave & 8721 \\
\hline allison.clark.net & Rob \& Jen's Genealogy Page & (*) 8476 \\
\hline helios.jicst.go.jp & Japan Information Center of Science and Technology & 8331 \\
\hline neoteny.eccosys.com & NetSurf mailing list & (*) 8036 \\
\hline
\end{tabular}

Table 4: Most-linked-to URLs

\section{Readability}

The UNIX utility style was used to assess the readability level of a subset of the HTML documents in our data set (approximately 150,000). We remove HTML markup before invoking style on each document. We do this for two reasons. First, style does not understand HTML, so the extra punctuation would confuse its analyzer. Second, breaking English text into sentences and sentence fragments can be tricky and we need to provide the style analyzer with some assistance. For example, it is not always 
clear when a bulleted list should be ignored, treated as a single long sentence, or treated as a list of individual sentences. When invoked on troff documents, style uses a set of heuristics to insert punctuation into text, using the markup to assist it [CHER81]. This information is then used by later passes of the analyzer to determine sentence and sentence fragment breaks. We use a similar set of heuristics to insert periods and commas into HTML documents as we strip out markup.

The numbers presented in Table 5 represent the scores of the different domains on the Kincaid readability test. Higher numbers represent more grammatical and lexical complexity. Lower numbers represent more simple structure and word choice. Documents with lower numbers are considered to be more "readable". The "other" domain is excluded because it represents extraordinarily diverse sources.

\begin{tabular}{|l|r|}
\hline Domain & Readability Score \\
\hline \hline com & 10.3 \\
\hline \hline edu & 11.0 \\
\hline gov & 10.0 \\
\hline \hline net & 12.3 \\
\hline \hline mil & 12.1 \\
\hline \hline org & 11.2 \\
\hline
\end{tabular}

Table 5: Average Readability

broken down by Domain

\section{Syntax Errors}

weblint was used to assess the syntactic correctness of a subset of the HTML documents in our data set (approximately 92,000). Figure 6 presents the top ten syntax errors ranked according to the percentage of documents in which they appear. (Note that "netscape-attribute"' is not necessarily an error, but rather indicates the percentage of documents using Netscape-specific extensions.) Observe that over $40 \%$ of the documents in our study contain at least one error. Descriptions of the errors appear in Table 6. 


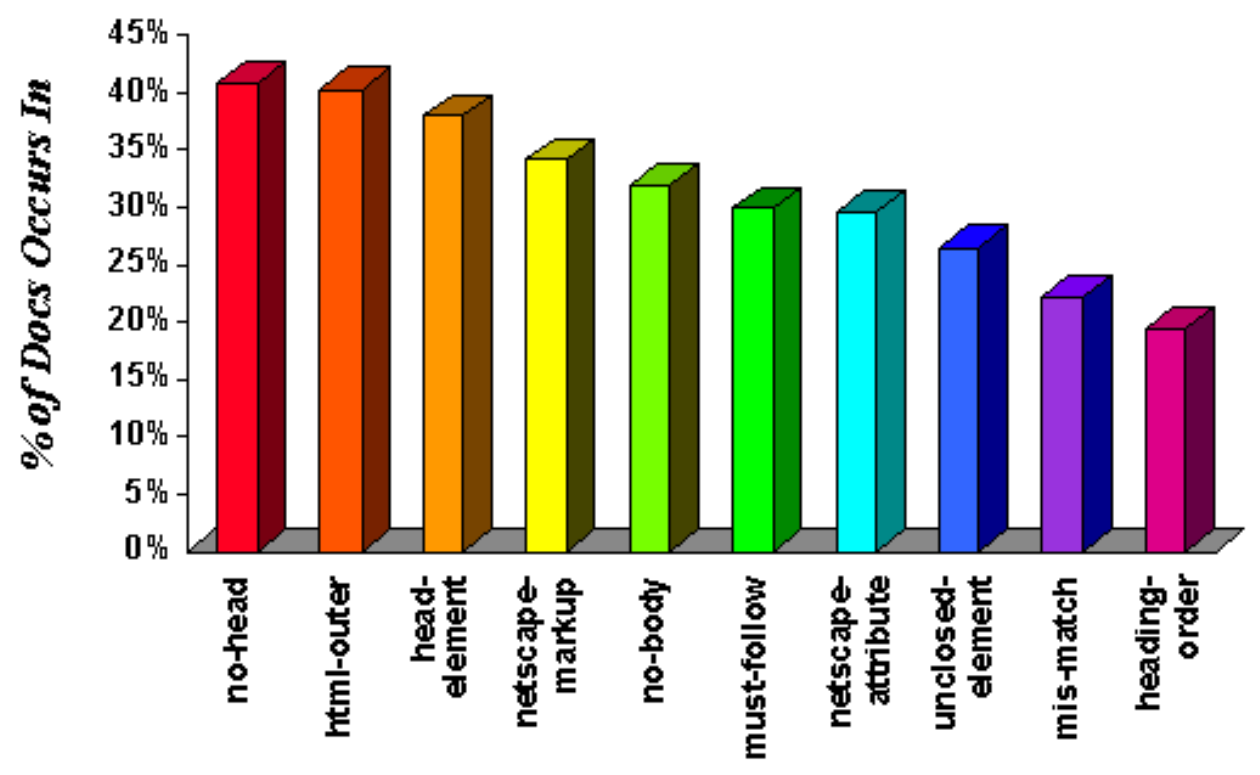

Figure 6: Ten Most Common Syntax Errors

\begin{tabular}{|c|c|}
\hline Error Name & Explanation \\
\hline html-outer & outer tags should be $\langle$ HTML $>$.. $</$ HTML $>$ \\
\hline no-head & missing 〈HEAD> \\
\hline head-element & $\begin{array}{l}\text { heading-only tag (TITLE, NEXTID, LINK, BASE, META) found outside of } \\
\text { heading }\end{array}$ \\
\hline no-body & missing <BODY> \\
\hline must-follow & required tag does not immediately follow another \\
\hline unclosed-element & unclosed elements (e.g., $<\mathrm{H} 1>\ldots$... \\
\hline netscape-markup & Netscape-specific tag \\
\hline empty-container & empty container element \\
\hline mis-match & mis-matched tag (e.g., $<\mathrm{H} 1>\ldots</ \mathrm{H} 2>$ ) \\
\hline heading-order & order of headings (e.g., $\langle\mathrm{H} 3>$ following $\langle\mathrm{H} 1>$ ) \\
\hline
\end{tabular}

Table 6: List of weblint Errors

\section{Conclusions}

We have reported the results of our examination of pages from the World Wide Web. Additional data not presented in the hardcopy version of this paper may be found at http://www.cs.berkeley.edu/ woodruff/inktomil

\section{Truisms}

There are two maxims which are particularly apropos of our experience. First, dealing with large data sets is difficult and time-consuming. None of the existing tools which we used scaled adequately to 
dealing with a data set on the order of millions of documents.

Second, we observed empirically that the Web changes exceptionally quickly. Many properties of the documents in our first data set have altered in the months since the data was collected. The largest document in our data set was 1.6Mbytes; we checked the current size of that same document. It has grown to 9Mbytes. As another example, many of the most popular URLs in the first data set no longer exist.

\section{Future Directions}

A longitudinal study examining trends would be extremely interesting. Our limited observation reveals that while certain charactertistics change fairly quickly (e.g., new features are introduced) others appear to change more slowly (e.g., average document size and reading level did not appear to change between the time periods we observed). One could also consider how the introduction of new tools impact these characteristics. For example, as authoring tools become more common, one could study their impact on the number and type of syntax errors.

Structural graph analysis has many applications in this area. In particular, analysis of the kind practiced by sociologists in structural network analysis [WASS94] promises insight. However, existing social network algorithms are several orders of magnitude more complex than is viable for a data set of this size. Significant work would have to be done to make such analysis feasible.

It would also be interesting to allow user-defined queries against the data set. The simplest functionality would be to allow a user to ascertain how a form-specified URL compared with the data set. A more interesting and complex interface would allow the user to define arbitrary queries on the data set.

\section{REFERENCES}

\section{[BOWE96]}

N. Bowers, “'Weblint Home Page (version 1.013),' Khoral Research, Inc., Albuquerque, NM, Jan. 1996. Available as http://www.khoral.com/staff/neilb/weblint.html.

[CATL95]

L. D. Catledge and J. E. Pitkow, "Characterizing Browsing Strategies in the World-Wide Web,"

Proc. 3rd Int. World Wide Web Conf., Darmstadt, Germany, Apr. 1995. Available as

http://www.igd.fhg.de/www/www95/proceedings/papers/80/userpatterns/UserPatterns.Paper4.form [CHER81]

L. L. Cherry, "Writing Tools - The STYLE and DICTION Programs,', Computer Science

Technical Report No. 91 (TM 79-1271-13), Bell Laboratories, Murray Hill, NJ, Feb. 1981.

Revised version reprinted as L. L. Cherry and W. Vesterman, "Writing Tools - The STYLE and DICTION Programs,' 4.4 BSD User's Supplementary Documents, Computer Science Research [CHI95] Group, Berkeley, CA, 1994.

E. H. Chi, 'Webspace Visualization,' The Geometry Center, Univ. of Minnesota, Minneapolis, MN. Available as http://www.geom.umn.edu/docs/weboogl/webspace/webspace.html.

\section{[COMM95]}

CommerceNet Consortium, “The CommerceNet/Nielsen Internet Demographics Survey,', Menlo Park, CA, 1995. Available as

http://www.commerce.net/information/surveys/execsum/exec_sum.html. 
[CONN95]

D. Connolly, "A Lexical Analyzer for HTML and Basic SGML," W3C Working Draft, World Wide Web Consortium, Cambridge, MA, Dec. 1995. Available as http://www.w3.org/pub/WWW/TR/.

[FIEL95]

R. Fielding, “Relative Uniform Resource Locators,” RFC 1808, June 1995. Available as http://www.cis.ohio-state.edu/htbin/rfc/rfc1808.

[FRYS94]

H. Frystyk and H. W. Lie, "Towards a Uniform Library of Common Code: A Presentation of the World Wide Web Library,"' Proc. 2nd Int. World Wide Web Conference, Chicago, IL, Oct. 1994. Available as http://www.ncsa.uiuc.edu/SDG/IT94/Proceedings/DDay/frystyk/LibraryPaper.html. [HANN95]

M. J. Hannah, "HTML Reference Manual," Sandia National Laboratories, Albuquerque, NM,

Dec. 1995. Available as http://www.sandia.gov/sci_compute/html_ref.html.

[KARP95]

R. Karpinski, "Hot Java Arrives: Sun Aims to Revolutionize the Web," InteractiveAge, May 22, 1995. Available as http://techweb.cmp.com/ia/15issue/15hotjava.html

[LYCO95]

Lycos, Inc., “The Lycos 250 and Hot Lists,” Pittsburgh, PA, Sep. 1995. Available as http://www.lycos.com/lists/index.html.

[MAUL94]

M. L. Mauldin and J. R. R. Leavitt, "Web Agent Related Research at the Center for Machine Translation," 1994 Meeting of the ACM Special Interest Group on Networked Information Discovery and Retrieval, McLean, VA, Aug. 1994. Available as http://fuzine.mt.cs.cmu.edu/mlm/signidr94.html, Carnegie Mellon Univ., Jul. 1994.

[MUKH95]

S. Mukherjea and J. D. Foley, "Visualizing the World-Wide Web with the Navigational View Builder,' Proc. 3rd Int. World Wide Web Conf., Darmstadt, Germany, Apr. 1995. Available as http://www.igd.fhg.de/www/www95/proceedings/papers/44/mukh/mukh.html.

[PIRO95]

P. Pirolli, J. Pitkow and R. Rao, "Silk from a Sow's Ear: Extracting Usable Structures from the Web," Xerox PARC, Palo Alto, CA, Nov. 1995. Submitted for publication.

[PITK94a]

J. E. Pitkow and K. Bharat, "WEBVIZ: A Tool for World Wide Web Access Log Visualization," Proc. 1st Int. World Wide Web Conf., Geneva, Switzerland, May 1994. Available as http://www1.cern.ch/WWW94/PrelimProcs.html.

[PITK94b]

J. E. Pitkow and M. M. Recker, "Results From The First World-Wide Web User Survey", Georgia Institute of Technology, Atlanta, GA, Jan. 1994. Available as http://www.gatech.edu/pitkow/survey/survey-1-1994/survey-paper.html.

[PITK95a]

J. E. Pitkow and M. M. Recker, "Using the Web as a Survey Tool: Results from the Second WWW User Survey,"' Proc. 3rd Int. World Wide Web Conf., Darmstadt, Germany, Apr. 1995. Available as http://www.igd.fhg.de/www/www95/proceedings/papers/79/survey/survey_2_paper.html. [PITK95b]

J. E. Pitkow and C. Kehoe, “The GVU Center's 3rd WWW User Survey,' Georgia Institute of Technology, Atlanta, GA, Apr. 1995. Available as 
http://www.cc.gatech.edu/gvu/user_surveys/survey-04-1995/.

[RISS95]

M. Rissa and C. Oy, “WWW User Survey Results,'” Helsinki, Finland, Feb. 1995. Available as http://www.mroy.fi/dec94.htm.

[WASS94]

S. Wasserman and K. Faust, 'SSocial Network Analysis: Methods and Applications,' Cambridge University Press, Cambridge, UK, 1994.

[YAHO95]

Yahoo, Inc., “Survey Says...” Mountain View, CA, Aug. 1995. Available as

http://www.yahoo.com/docs/survey/first.html and http://www.yahoo.com/docs/survey/index.html. 